\title{
ENHANCEMENT OF DISSOLUTION CHARECTERISTICS AND ORAL BIOAVAILABILITY OF TACROLIMUS FROM PRONIOSOMAL DRUG DELIVERY SYSTEM
}

\author{
Pradeep Kumar Yennamameni ${ }^{1 *}$, Raju Jukanti ${ }^{2}$, Subba Rao D ${ }^{3}$, Vamshi Krishna M $^{1}$ \\ ${ }^{1}$ Department of Pharmaceutics, Jangaon Institute of Pharmaceutical sciences, Jangaon, Telangana, India. \\ ${ }^{2}$ Telangana Drugs Control Administration, Karimnagar, Telangana, India. \\ ${ }^{3}$ Department of Chemical Engineering, JNTU ananthapur, Ananthapuramu, Andhra Pradesh, India.
}

\begin{abstract}
*Corresponding Author Email: pradppharma@yahoo.com
ABSTRACT

The current research was designed to improve the oral delivery of tacrolimus by loading into maltodextrin based proniosome powders. proniosome powders proved to be the potential carriers for efficient oral delivery of lipophilic or amphiphilic drugs. These 'proniosomes' minimize problems of niosome physical stability such as aggregation, fusion and leaking, and provide additional convenience in transportation, distribution, storage, and dosing. The proniosome powders were fabricated by various ratios of span 60 and cholesterol and evaluated for micromeritic properties and the results indicates adequate micrometric properties. The optimized formulation showed smaller vesicle size, high surface charge and entrapment efficiency. The optimized proniosome formulation was evaluated by optical and scanning electron microscopy, FT-IR, differential scanning calorimetry, and powder X-ray diffraction to understand the morphological, solid state properties of the drug reveal the absence of chemical interaction, drug transformation from crystalline to amorphous and molecular state. Multi media dissolution profiles [at $\mathrm{pH}$ 1.2, 4.5 and 6.8] were carried out to demonstrate improved dissolution characteristics compared to pure drug.
\end{abstract}

\section{KEY WORDS}

Proniosomes, Maltodextrin, Span 60, Tacrolimus, Dissolution

\section{Introduction:}

A number of vesicular drug delivery systems have emerged encompassing various routes of administration to achieve controlled and targeted drug delivery such as liposome, niosome, transferosomes, ethosomes and proniosome were developed. Provesicular concept has evolved to resolve the stability issue pertaining to the conventional vesicular systems i.e. liposomes and niosomes. In recent years, vesicles have become the vehicle of choice in drug delivery. Lipid vesicles were found to be of value in immunology, membrane biology, diagnostic techniques and most recently, genetic engineering. Vesicles can play a major role in modeling biological membranes, and in the transport and targeting of active agents. Encapsulation of a drug in vesicular structures can be predicted to prolong the existence of the drug in systemic circulation and perhaps, reduces the toxicity if selective uptake can be achieved ${ }^{1}$.

Proniosomes are vesicular systems, in which the vesicles are made up of non-ionic based surfactants, cholesterol and other additives which may be hydrated immediately before use to yield aqueous niosome dispersions. In the current work, proniosomal formulation for tacrolimus was developed using simple slurry method.

\section{Materials and Methods:}

\section{Materials:}

Tacrolimus was gifted from Mylan Laboratories Limited Hyderabad, India, Maltodextrin was gifted by Sigma Aldrich Chemicals, Hyderabad, Cholesterol and 
Span 60 was purchased from SD Fine Chemicals, Mumbai. Methanol and Chloroform were purchased from Merck Specialties Pvt. Ltd, Mumbai.

\section{Solubility studies of Tacrolimus}

An excess amount of tacrolimus was added to each vial containing $1 \mathrm{~mL}$ of the vehicle. After sealing, the mixture was vortexed using a vortex mixer at a maximum speed for $10 \mathrm{~min}$ in order to facilitate proper mixing of tacrolimus with the vehicle. Mixtures were then shaken in an orbital shaker maintained at room temperature until equilibrium (24 h). Mixtures were centrifuged at 15,000 rpm for $10 \mathrm{~min}$, and the resulting supernatant was filtered through the Whatman filter paper. The filtrate was quantified by the developed HPLC method using methanol and water (60:40 \% v/v) as mobile phase and $294 \mathrm{~nm}$ wavelength was used.

\section{Formulation of Proniosomal Powder: ${ }^{3,4,5}$}

Proniosome powders were prepared by using slurry method. The composition of different proniosomal formulations is represented in Table 1.

Emulsifier/lipid mixture comprising of span 60, cholesterol and drug as per formulation ratios were accurately weighed and dissolved in $20 \mathrm{~mL}$ of solvent mixture containing chloroform and methanol (2:1). The above solvent solution was transferred into a $250 \mathrm{~mL}$ round bottom flask and required amount of maltodextrin was added to form slurry. The flask was attached to a rotary flash evaporator (Hei-VAP advantage/561-0300, Heidolph, Germany) and the organic solvent was evaporated under reduced pressure at a temperature of $45 \pm 2^{\circ} \mathrm{C}$. After ensuring the complete removal of solvent, the resultant powders were further dried overnight in a vacuum oven at room temperature so as to obtain dry, freeflowing product. The obtained proniosome powders were stored in a tightly closed container at $2-8^{\circ} \mathrm{C}$ for further characterization.

Table 1: Formulation development of Tacrolimus Proniosomes

\begin{tabular}{llllllll}
\hline Ingredients & TPN1 & TPN2 & TPN3 & TPN4 & TP5 & TPN6 & TPN7 \\
\hline Drug (mg) & 5.0 & 5.0 & 5.0 & 5.0 & 5.0 & 5.0 & 5.0 \\
Maltodextrin (mg) & 150 & 150 & 150 & 150 & 150 & 150 & 150 \\
$\begin{array}{l}\text { Span 60(mg) } \\
\text { Cholesterol(mg) }\end{array}$ & 175 & 150 & 125 & 100 & 75 & 50 & 25 \\
$\begin{array}{l}\text { (2:1) Chloroform } \\
\text { and Methanol (mL) }\end{array}$ & 20 & 50 & 75 & 100 & 125 & 150 & 175 \\
\hline
\end{tabular}

\section{Characterization of proniosome powders Morphological evaluation}

The morphology of the proniosomes was evaluated by optical microscopy. The proniosome powder was placed on a cavity glass slide and few $\mathrm{mL}$ of water was added drop wise along the side of the cover slip. The formation of vesicles was monitored through an optical microscope (METZER optical instrument) and photographs were taken.

\section{Micromeritic properties}

The flow properties of powder are plays an important role in handling and processing operations. The flow properties were studied through measuring the Angle of repose, Carr's compressibility index and Hausner's ratio. The conventional fixed funnel method was used for angle of repose determination. The bulk and tapped density of the proniosome powders evaluated for calculating the Carr's compressibility index and Hausner's ratio.

\section{Re-dispersible properties}

The number of vesicles formed after hydration is one of the important parameter to evaluate the proniosome powder. The proniosome powder was hydration with $\mathrm{pH} 6.8$ phosphate buffer and the formed niosomes were counted by optical microscope using a haemocytometer. The niosomes in 80 small squares were counted and calculated by using the following formula. 
Total number of niosomes per cubic millimetre $=$ Total number of niosomes counted $x$ Dilution factor $x$ 4000/ Total number of squares counted.

\section{Determination of vesicle size, Zeta Potential}

The proniosomal powders were hydrated with phosphate buffer ( $\mathrm{pH}$ 6.8) and subjected to bath sonication for $3 \mathrm{~min}$ and the resultant dispersion was used for the determination of size, zeta potential. The mean size, size distribution and zeta potential (ZP) of proniosomes was determined photon correlation spectroscopy using Zetasizer NanoZS90. Each sample was diluted to a suitable concentration with phosphate buffer $\mathrm{pH}$ 6.8. Size analysis was performed at $25{ }^{\circ} \mathrm{C}$ with an angle of detection of $90^{\circ} \mathrm{C}$. Size, polydispersity index of proniosomes and their mean zeta potential values $( \pm S D$ ) were obtained from the instrument.

\section{Percentage Drug Entrapment}

The percentage drug entrapped (PDE) was determined by Ultra-centrifugation. The liposomal formulations were subjected for ultracentrifugation (ultraCentrifuge - Remi laboratories, Mumbai, India) at 5000 rpm for $15 \mathrm{~min}$ in an ultracentrifuge in order to separate the entrapped drug from the free drug. Then the clear supernatant was separated and analyzed for drug content after appropriate dilution by HPLC. This indicates amount of free drug. The liposomal pellet was redispersed in Methanol and analyzed for drug content after appropriate dilution by HPLC at $294 \mathrm{~nm}$. This indicates amount of drug entrapped. The entrapment capacity of liposomes was calculated as follows

$\mathrm{PDE}=[(\mathrm{T}-\mathrm{C}) / \mathrm{T}] \times 100$

Where $T$ is the total amount of drug that is detected both in the supernatant and sediment, and $C$ is the amount of drug detected only in the supernatant [1-3].

\section{In-vitro drug release study}

In vitro dissolution study of proniosomal powders and pure drug was performed using USP type II (paddle) apparatus in multimedia dissolution media of $\mathrm{pH} 1.2$, pH 4.5 and pH6.8 to maintain sink conditions [4,5]. The volume of dissolution medium used was $900 \mathrm{~mL}$ and maintained at a temperature of $37 \pm 1$ o $C$ with $50 \mathrm{rpm}$ of paddle speed set at throughout the experiment. 5 $\mathrm{mL}$ of aliquot of was collected at predetermined time intervals at 30,60, 90, 120min and replaced with fresh dissolution medium to maintain constant volume ${ }^{40}$.
The samples were filtered by passing through $0.45 \mu \mathrm{m}$ PVDF membrane filter (Millipore) and analyzed by HPLC at $294 \mathrm{~nm}$. Cumulative \% of drug released was calculated and plotted against time $(\mathrm{t})$.

\section{HPLC analysis of Tacrolimus}

Assay of tacrolimus samples were analysed by using HPLC method and HPLC method adapted from USP of tacrolimus [20]. The HPLC system consisted of a vaccum degasser, photodiode array detector. LC mode and 4.6-mm X 15-cm; 3- $\mathrm{mm}$ packing L1 column used for the chromatographic separation. The mobile phase was Solution was used methanol and water (60:40 \% $\mathrm{v} / \mathrm{v}$ ). pumped at a constant flow rate of $1.5 \mathrm{~mL} / \mathrm{min}$. the eluent was monitored using detector at a wavelength of $294 \mathrm{~nm}$. The column was maintained at $60^{\circ} \mathrm{C}$, auto sampler at $4^{\circ}$ and injection volume of $20 \mu \mathrm{L}$ was injected. The mobile phase was filtered through $0.45 \mu \mathrm{m}$ PTFE filter. Under these conditions, the retention time $\left(t_{R}\right)$ of Tacrolimus was approximately $5.3 \mathrm{~min}$.

\section{Solid State Characterization Studies [6-8]: Differential scanning calorimetry (DSC)}

The DSC analysis was used to evaluate the molecular state of the encapsulated drug in optimized proniosomal formulation(TPN4), Pure drug, maltodextrin. Each sample was placed in an aluminum pan and then crimped with an aluminum cover. The DSC curves of the samples were obtained by a differential scanning calorimeter (DSC-6300, SIIO, Japan). The heating and cooling rates were $10 \circ \mathrm{C} / \mathrm{min}$ and $250 \circ \mathrm{C} / \mathrm{min}$, respectively. All measurements were performed over $0-400^{\circ} \mathrm{C}$ under a nitrogen purge at 50 $\mathrm{mL} / \mathrm{min}$.

\section{Powder X-ray diffractometry (PXRD)}

Every crystalline substance gives a pattern; the same substance always gives the same pattern; and in a mixture of substances each produces its pattern independently of the others."

crystalline properties of the optimized proniosome powder formulation, pure drug and maltodextrin were analysed by using X-ray diffractometer (Stereoscans S120, Cambridge, UK). The measuring conditions were as follows: CuKa radiation, nickel filtered; graphite monochromator; $45 \mathrm{kV}$ voltage; and $40 \mathrm{~mA}$ currents with $X^{\prime}$ celerator detector. All samples were run at $1^{0}$ (2ө) $\min ^{-1} 1$ from $3^{\circ}$ to $45^{\circ}(2 \theta)$. 


\section{Scanning electron microscopy (SEM)}

The surface morphology and the internal textures of the pure drug, maltodextrin and proniosome powder were investigated by scanning electron microscope. Samples were fixed on a brass stub using double sided adhesive tape and were made electrically conductive by coating with a thin layer of gold and SEM images were recorded at $15 \mathrm{kev}$ accelerating voltage.

\section{Fourier transform infrared (FT-IR) spectroscopy}

Infrared spectra of pure drug, maltodextrin, non-ionic surfactant and optimized proniosome powder formulation were obtained using FT-IR spectrophotometer (Bruker, Alpha-T, Lab India) by the conventional $\mathrm{KBr}$ pellet method.

\section{In-vivo or Ex-vivo permeation studies:}

\section{Permeation data analysis}

The cumulative amount of drug permeated (Q) was plotted against time. The steady state flux (Jss) was calculated from the slope of linear portion of the cumulative amount permeated per unit area vs. time plot. The permeability coefficient (Kp) of the drug through intestine was calculated by dividing steady state flux with initial concentration of tacrolimus in donor compartment [9]. The enhancement ratio (ER) was calculated by using the following equation: $E R=$ Jss of proniosome formulation/Jss of control.

\section{In situ, intestinal absorption study [10-12]}

The in situ intestine absorption of tacrolimus from proniosome formulations was assessed by adapting single-pass perfusion method. Male wistar rats weighing between 180-200 gm used in the study were obtained from Mahaveera Enterprises (146-CPCSEA no: 199; Hyderabad, India). The animals were housed in separate cages in a clean room and maintained under controlled condition of temperature and the rats had free access to food and water. The study was conducted with the prior approval of Institutional Animal Ethical Committee. Euthanasia and disposal of carcass was in accordance of the guidelines. Before perfusion experiments, the rats fasted overnight with free access to water was anesthetized by thiopental sodium injection ( $60 \mathrm{mg} / \mathrm{kg}$ body weight) administered via intraperitoneal route and placed on a thermostatic surface to maintain body temperature. Under anesthesia, an incision was made through a midline to expose the abdominal content. The lower part of the small intestine segment used for perfusion was exposed and semi-circular incisions were made on both ends and cannulated with PE tubing followed by ligation with silk suture. After cannulation, the surgical area was covered with cotton soaked in physiological saline $\left(37^{\circ} \mathrm{C}\right)$ throughout the experimentation. To remove the adhered mucosal contents, the intestine segment was flushed with phosphate buffered saline (PBS) $\left(\mathrm{pH} 7.4\right.$ at $37^{\circ} \mathrm{C}$ ) and stabilized by perfusing the blank PBS for $15 \mathrm{~min}$. The perfusates prepared by dispersing proniosome powders (TN4P and control formulation (processed without phospholipids and span 80 ) equivalent to $5 \mathrm{mg}$ of tacrolimus containing phenol red $(7.5 \mu \mathrm{g} / \mathrm{mL})$ in PBS were passed at a steady flow rate of $0.2 \mathrm{~mL} / \mathrm{min}$ (NE-1600, New Era Syringe Pumps, USA) and the perfusate was collected for every $15 \mathrm{~min}$. At the end of the perfusion $(90 \mathrm{~min}$ ) the circumference and length of the perfused intestine was measured and the samples were stored at $-20^{\circ} \mathrm{C}$ until further analysis by HPLC. Each experiment was performed in triplicate. Prior to analysis, the perfusate samples were allowed to thaw, deproteinized with methanol, centrifuged and the drug content in the supernatant was quantified for tacrolimus by HPLC.

\section{Stability studies}

The optimized proniosomal formulation was filled in glass vials and charged into stability for 180days at controlled room temperature and in refrigerator (2 $\left.8^{\circ} \mathrm{C}\right)$. Samples were withdrawn at predefined time intervals (Initial, 30, 60, 90, 120 and 180days) and hydrated with phosphate buffered saline $\mathrm{pH}(6.8)$ and observed under optical microscope for any sign of drug morphology. Further the samples were analysed for percentage potency of tacrolimus present optimised formulation and compared to the initial evaluated results to establish the stability of proniosomal formulation.

\section{Results and Discussions}

Preparation and physico-chemical evaluation of proniosome powders

Maltodextrin had porous structure and high surface area and easy adjustment of amount of carrier required to support the surfactant and several researchers reported that Maltodextrin based 
proniosomes proved to be the potential carriers for efficient oral delivery poorly soluble drugs.

Tacrolimus loaded maltodextrin based proniosomes with span 60 as nonionic surfactant prepared by a slurry reported in the literature. Colloidal carrier drug delivery approach of proniosome approach has resolved many stability issues pertaining to aqueous niosome dispersions. The advantages, noisome dispersions suffer from stability problems like aggregation, hydrolysis, drug leakage and production scale up. In this perspective for improve the stability of the vesicular systems several strategies have been used. To improve the stability and entrapment efficiency of vesicular formulations cholesterol is the first choice of additive used as a structural lipid. The concentration of nonionic surfactant and cholesterol will influence the morphology and stability of the vesicular systems like niosomes drug delivery system. The various ratios of cholesterol and span 60 were evaluated for the optimum composition of span 60 to cholesterol ratio by keeping the total lipid constant.
The phase transition temperatures of various spans are, span 20 is $16^{\circ} \mathrm{C}$, span $40^{\circ} \mathrm{C}$ is $42^{\circ} \mathrm{C}$, span 60 is $53^{\circ} \mathrm{C}$ and span 80 is $12{ }^{\circ} \mathrm{C}$, the span 60 having high phase transition temperature and span 80 having the low phase transition temperature. Highest phase transition temperature of span having high entrapment could be observed as reported in the literature. Span 60 was selected as choice of nonionic surfactant due to the high phase transition temperature, it interns led's to high entrapment efficiency, to facilitate stable vesicle formation and to improve the oral delivery of tacrolimus from proniosomes.

Morphological evaluation of prepared proniosome powders by optical microscope

The niosomes formed from proniosome powder after hydration and gentle shaking with water as shown in Figure 1. multilamellar vesicles are formed after gentle agitation with and all vesicles are in spherical shape. The morphology of the proniosomes was evaluated by optical microscopy.
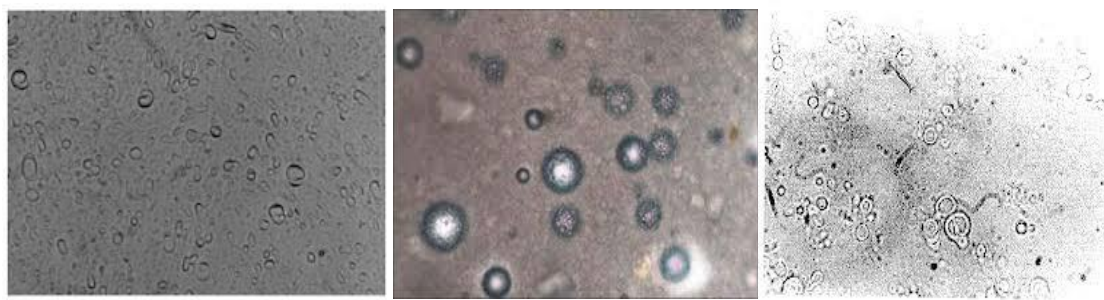

Fig. 1. Optical microphotographs showing (A) proniosome powder, (B) formation of vesicles on maltodextrin after hydration with phosphate buffer (pH 6.8), (C) Niosome dispersion from proniosome powder (TPN4) upon gentle agitation.

\section{Micromeritic properties of proniosome powders}

The micromeritics of the proniosome powders is vital in handling and processing operations because the dose uniformity and ease of filling into container is dictated by the powder flow properties. In general, three types of flow measurements can be used to evaluate the nature of powder flow i.e. angle of repose; Carr's index and Hausner's ratio and the results were depicted in Table 2 . The smaller the value of angle of repose, lesser the internal friction or cohesion between the particles and greater the flow characteristics and vice-versa. It is apparent from the results that small angle of repose $\left(<20^{\circ}\right)$ assure good flow properties for proniosome powder formulations. In addition to angle of repose, Carr's index and Hausner's ratio were also less than 17 and 1.25 respectively ensuring acceptable flow for proniosomes powder formulations and results are reported in Table 2. 
Table 2: Micromeritic properties of various proniosome powder formulations.

\begin{tabular}{llll}
\hline Formulation & $\begin{array}{l}\text { Angle of } \\
\text { repose }(\theta)\end{array}$ & $\begin{array}{l}\text { Compressibility } \\
\text { index }\end{array}$ & $\begin{array}{l}\text { Hausner's } \\
\text { ratio }\end{array}$ \\
\hline TPN1 & $16.8 \pm 0.21$ & $10.8 \pm 0.08$ & $1.09 \pm 0.15$ \\
TPN2 & $19.2 \pm 0.23$ & $11.4 \pm 0.06$ & $1.18 \pm 0.19$ \\
TPN3 & $19.8 \pm 0.32$ & $15.5 \pm 0.25$ & $1.22 \pm 0.13$ \\
TPN4 & $18.1 \pm 0.35$ & $16.2 \pm 0.37$ & $1.17 \pm 0.21$ \\
TPN5 & $19.3 \pm 0.27$ & $15.7 \pm 0.28$ & $1.23 \pm 0.12$ \\
TPN6 & $19.6 \pm 0.14$ & $16.8 \pm 0.26$ & $1.20 \pm 0.14$ \\
TPN7 & $19.2 \pm 0.26$ & $16.4 \pm 0.41$ & $1.17 \pm 0.09$ \\
\hline
\end{tabular}

\section{Number of vesicles per cubic millimetre}

The hyderated niosomes were counted by optical microscope using a haemocytometer. The niosomes in 80 small squares were counted, calculated and tabulated table 3 . Total number of niosomes per cubic millimetre

Determination of vesicle size, Zeta potential and Entrapment efficiency and No. of vesicles per $\mathrm{mm}^{3}$ :

Several researchers are reported that the vesicle size and size distribution of proniosomes is most important parameter, which needs to monitor during proniosomes preparation for its best performance.

Proniosomal sample was placed under digital microscope (Metzer, India) and hydrated with milli Q water. Then formation of vesicle was observed within the niosomal dispersion. Results of average vesicle size and distribution were calculated for count and distribution. The maximum benefit from the proniosome formulations can be speculated when abundant numbers of vesicles are formed after hydration in the gastrointestinal tract. Among all the formulations, the proniosome formulation containing span 60 and cholesterol at a ratio of 1:1 (TPN4) has exhibited good number of vesicles which is min well correlation with the size and entrapment efficiency results.

Vesicle size and size distribution is an important parameter for the vesicular systems [34]. The mean size of the vesicles was in the range of $200-480 \mathrm{~nm}$. Small value of polydispersity index $(\mathrm{PI})(<0.1)$ indicates a homogenous population, while a $\mathrm{PI}(>0.3)$ indicates a higher heterogeneity. The zeta potential and entrapment efficiency of the formulations was between -26.5 and $-36.5 \mathrm{mV}$ and 60- 92\%, respectively. Determination of entrapment efficiency is an important parameter in case of proniosome as it majorly effects the drug release. Entrapment efficiency is expressed as the fraction of drug incorporated into proniosome relative to total amount of drug used. The size and surface charge of the vesicles seems to be dependent on the cholesterol concentration. We could notice small sized vesicles with high zeta potential when equimolar ratio of formulation containing span 60 and cholesterol (TPN4) and also.

Table 3: Physico-chemical characterization of various proniosome formulations

\begin{tabular}{lllllll}
\hline $\begin{array}{l}\text { S. } \\
\text { No }\end{array}$ & Formulation & $\begin{array}{l}\text { size } \\
(\mathbf{n m})\end{array}$ & PI & $\begin{array}{l}\text { Zeta } \\
\text { potential } \\
(\mathbf{m V})\end{array}$ & $\begin{array}{l}\text { Entrapment } \\
\text { efficiency } \pm \text { SD } \\
(\%)\end{array}$ & $\begin{array}{l}\text { No. of } \\
\text { vesicles } \\
\text { per } \mathbf{~ m m}^{\mathbf{3}} \mathbf{x} \\
\mathbf{1 0}^{\mathbf{3}}\end{array}$ \\
\hline 1 & TPN1 & $434 \pm 23$ & 0.356 & $33.1 \pm 4.8$ & $94.9 \pm 0.244$ & 3.02 \\
2 & TPN2 & $343 \pm 12$ & 0.281 & $27.7 \pm 5.2$ & $85.12 \pm 1.48$ & 3.81 \\
3 & TPN3 & $265 \pm 07$ & 0.278 & $35.3 \pm 3.1$ & $91.02 \pm 0.63$ & 3.21 \\
4 & TPN4 & $206 \pm 12$ & 0.178 & $41.4 \pm 2.3$ & $96.5 \pm 0.205$ & 4.14 \\
5 & TPN5 & $334 \pm 23$ & 0.294 & $34.1 \pm 3.5$ & $92.7 \pm 0.249$ & 3.23 \\
6 & TPN6 & $412 \pm 16$ & 0.312 & $28.0 \pm 4.2$ & $94.1 \pm 0.509$ & 3.91 \\
7 & TPN7 & $480 \pm 21$ & 0.357 & $26.5 \pm 2.6$ & $88.1 \pm 2.19$ & 4.21 \\
\hline
\end{tabular}




\section{In vitro studies:}

The dissolution profiles of proniosomes formulation were shown in figure $2 \mathrm{~B}$. The amount of tacrolimus released from proniosomes was ranging between 50 to $85 \%$ in 90 min and was higher compared to control (30 $\%)$ at all multimedia dissolution. The dissolution efficiency of insoluble drug tacrolimus has been significantly improved when encapsulated in proniosomes. This might be due to the enhanced solubility of tacrolimus by span 60 molecules. However, we could not notice any remarkable change in the dissolution behavior with different proniosome formulations. Interestingly, the proniosome formulation (TPN4) promoted higher dissolution of tacrolimus compared to other formulations. The improved dissolution of the drug from the proniosome powder formulations might be due to the altered physical state of the drug entrapped within the niosome bilayer and enhanced effective surface area available for dissolution medium. From the above results TPN4 formulation has selected as optimized one which is used for further study. The dissolution profiles and cumulative dissolution of tacrolimus from proniosome powder formulations (TPN1-TPN7) and control (pure drug) in $\mathrm{pH} 1.2 \mathrm{pH} 4.5$ and $\mathrm{pH} 6.8$ dissolution media are depicted in Fig. 2 And Table 4.

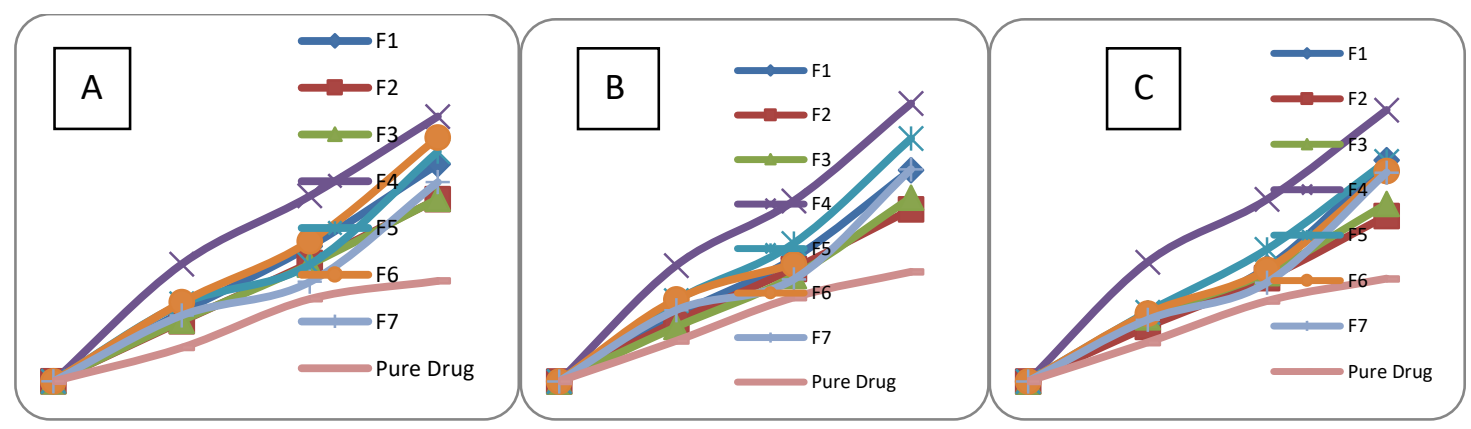

Figure 2: Dissolution profiles of tacrolimus from proniosome formulations in $\mathrm{pH} 1.2(\mathrm{~A}), \mathrm{pH} 4.5(\mathrm{~B})$ and $\mathrm{pH} 6.8(\mathrm{C})$ dissolution media

Table 4: Cumulative \% Drug Release from various Proniosome formulations of Tacrolimus after 90 min (TPN1-TPN7 \& Pure Drug)

\begin{tabular}{|l|l|l|l|l|}
\hline $\begin{array}{l}\text { S. } \\
\text { No }\end{array}$ & Formulation & $\begin{array}{l}\text { Cumulative } \\
\text { \% drug } \\
\text { release in pH } \\
\mathbf{1 . 2}\end{array}$ & $\begin{array}{l}\text { Cumulative \% } \\
\text { drug release } \\
\text { in pH 4.5 }\end{array}$ & $\begin{array}{l}\text { Cumulative \% } \\
\text { drug release } \\
\text { in pH 6.8 }\end{array}$ \\
\hline 1 & TPN1 & 66.0 & 65.3 & 68.3 \\
\hline 2 & TPN2 & 55.4 & 53.1 & 51.1 \\
\hline 3 & TPN3 & 55.5 & 56.8 & 54.8 \\
\hline 4 & TPN4 & 80.5 & 85.8 & 83.8 \\
\hline 5 & TPN5 & 69.8 & 75.0 & 68.0 \\
\hline 6 & TPN6 & 74.1 & 68.0 & 65.0 \\
\hline 7 & TPN7 & 60.5 & 65.5 & 64.5 \\
\hline 8 & Pure Drug & 30.5 & 33.8 & 31.7 \\
\hline
\end{tabular}

\section{Solid state characterization}

Scanning electron microscopy, differential scanning calorimetry, X-ray powder diffraction and fourier transform infrared spectroscopy used evaluation of the molecular interactions between drug and carrier. The thermotropic behavior and the physical state of the drug in proniosome powders were evaluated by performing DSC analysis. The DSC thermograms of 
tacrolimus, maltodextrin and proniosome formulation (TPN4) were recorded (Fig. 3). The maltodextrin used as a carrier exhibited a difused peak at $359.0^{\circ} \mathrm{C}$ corresponding to its melting point. The absence of conspicuous peak in proniosome formulation over the melting range of API unravels the transformation of the physical state of the drug (crystalline to amorphous) which was further confirmed by PXRD analysis.

The PXRD patterns of tacrolimus, maltodextrin and proniosomal powder were represented in figure 4 . The pure drug showed numerous characteristic high intensity diffraction peaks at $2 \theta$ of $8.8,11.08,13.97$, 17.73 and 19.34 demonstrating the crystalline nature of the drug. The absence or reduced intensities of characteristic tacrolimus peaks in proniosome formulation suggest the change in physical state i.e. amorphization of drug.

Figure 5 illustrates the FT-IR spectra of tacrolimus, maltodextrin and proniosome formulation (TPN4). The pure drug tacrolimus exhibits characteristic peaks bands at 1713, 1248 and $709 \mathrm{~cm}-1$, as well as the benzene ring bands at 1450, 1500 and $1600 \mathrm{~cm}-1$. The peaks at 1713, 1248, 709, 1450, 1500 and 1600 $\mathrm{cm}-1$ were disappeared in proniosome formulation and the intensity of peaks at 1713, 1248 and $709 \mathrm{~cm}-1$ was reduced. However, no additional peaks in proniosome formulation indicate the absence of chemical interaction between the drug and formulation ingredients.

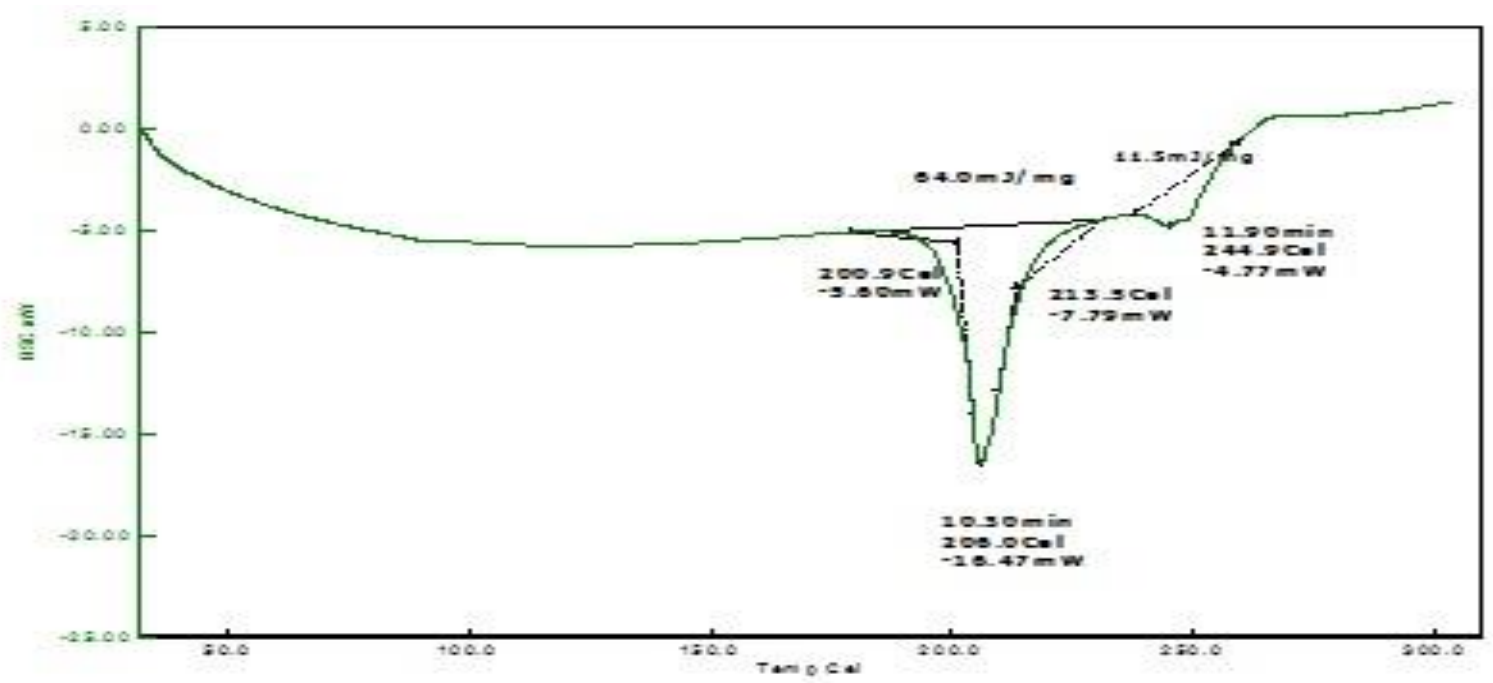

Figure 3a : DSC thermogram of Tacrolimus

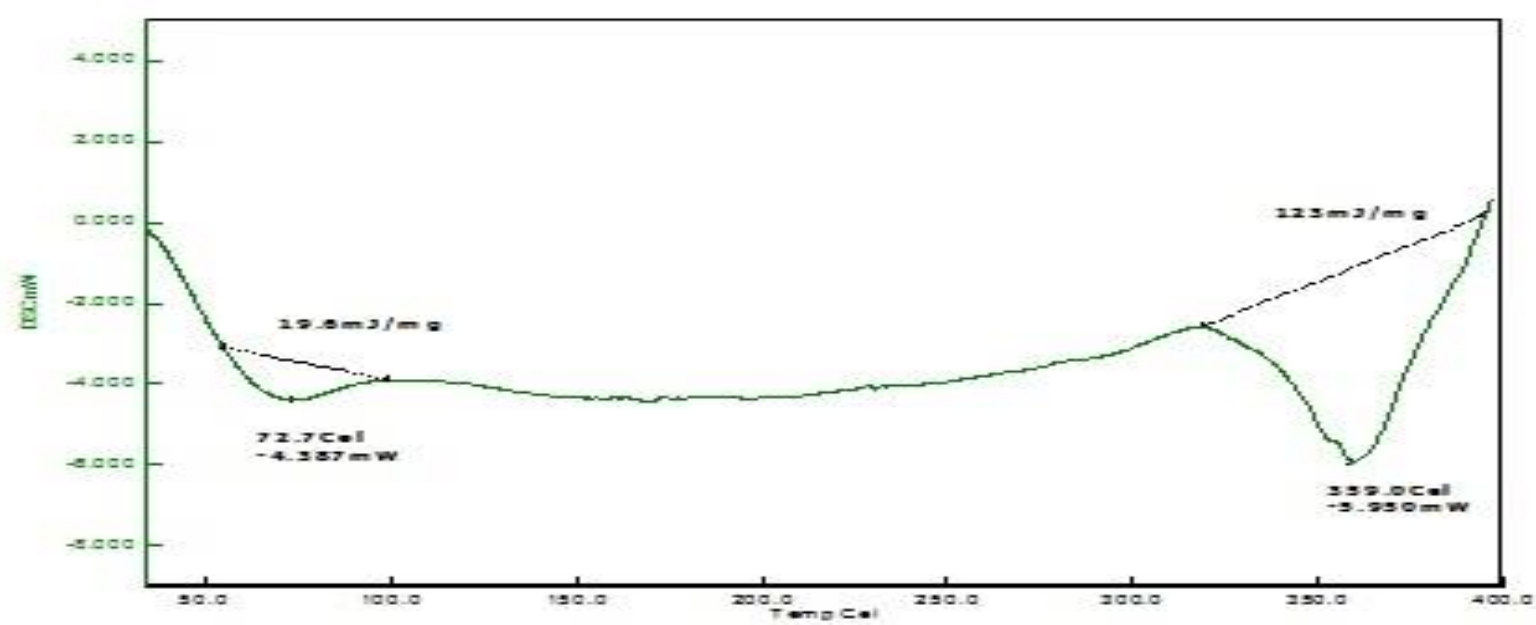

Figure 3b : DSC of Maltodextrin 


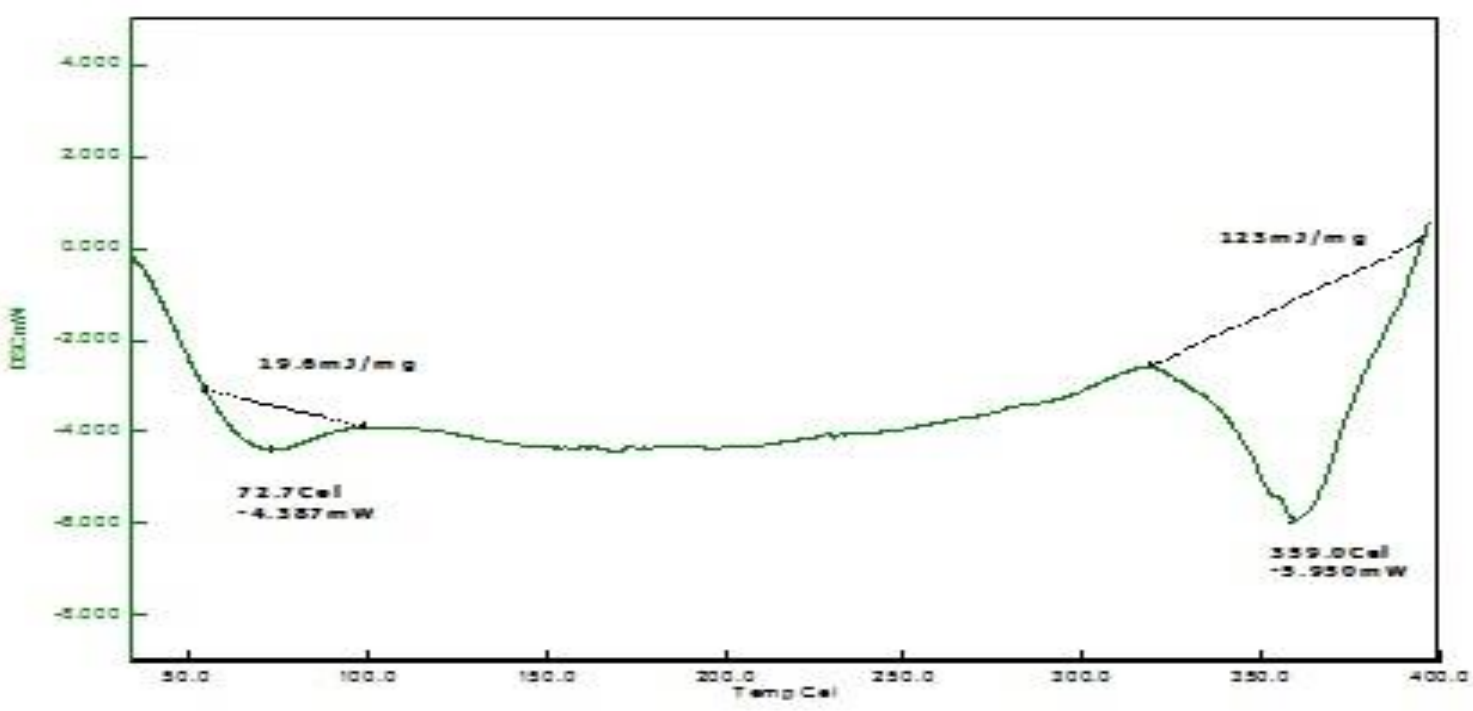

Figure 3c: DSC of Optimized Formulation TPN4

Powder X-Ray Diffraction studies:

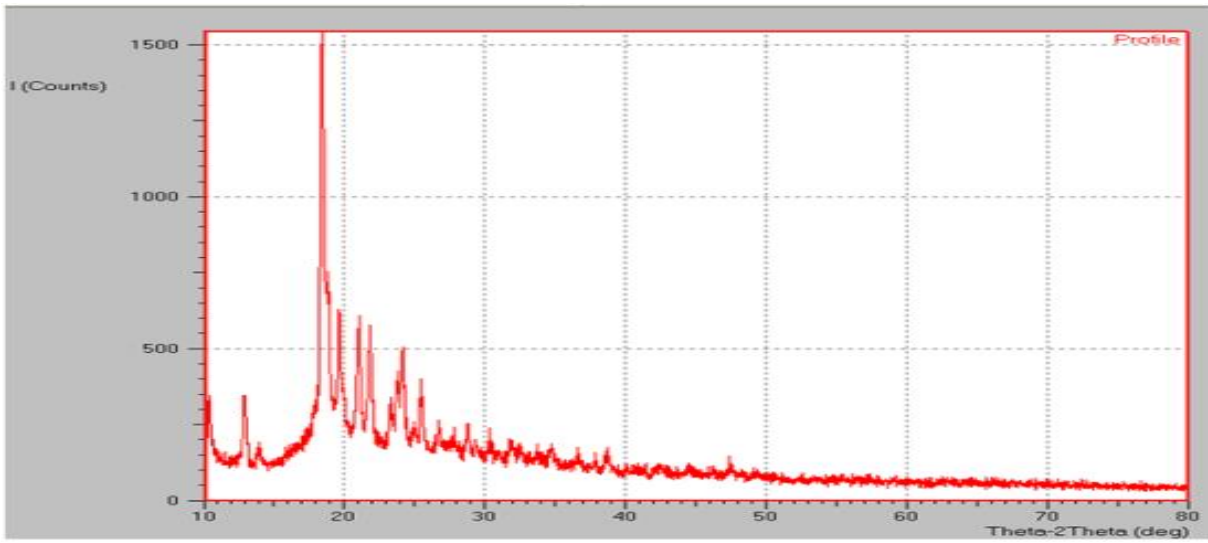

Figure 4a: PXRD of Tacrolimus

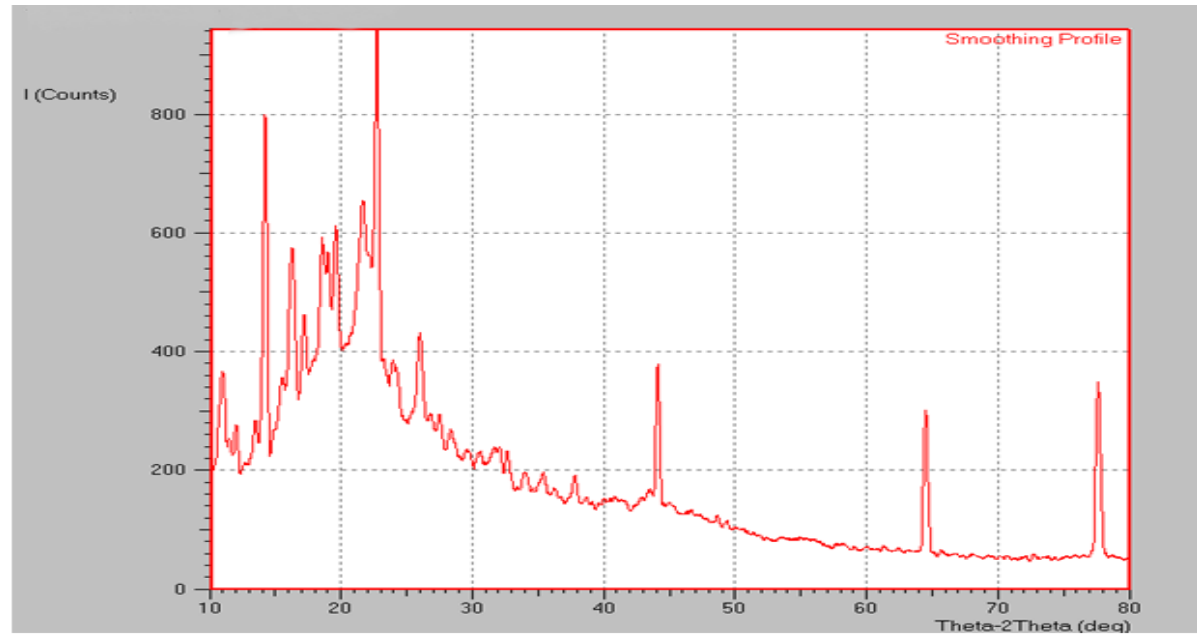

Figure 4b: PXRD of Optimized formulation TPN4 
FTIR:

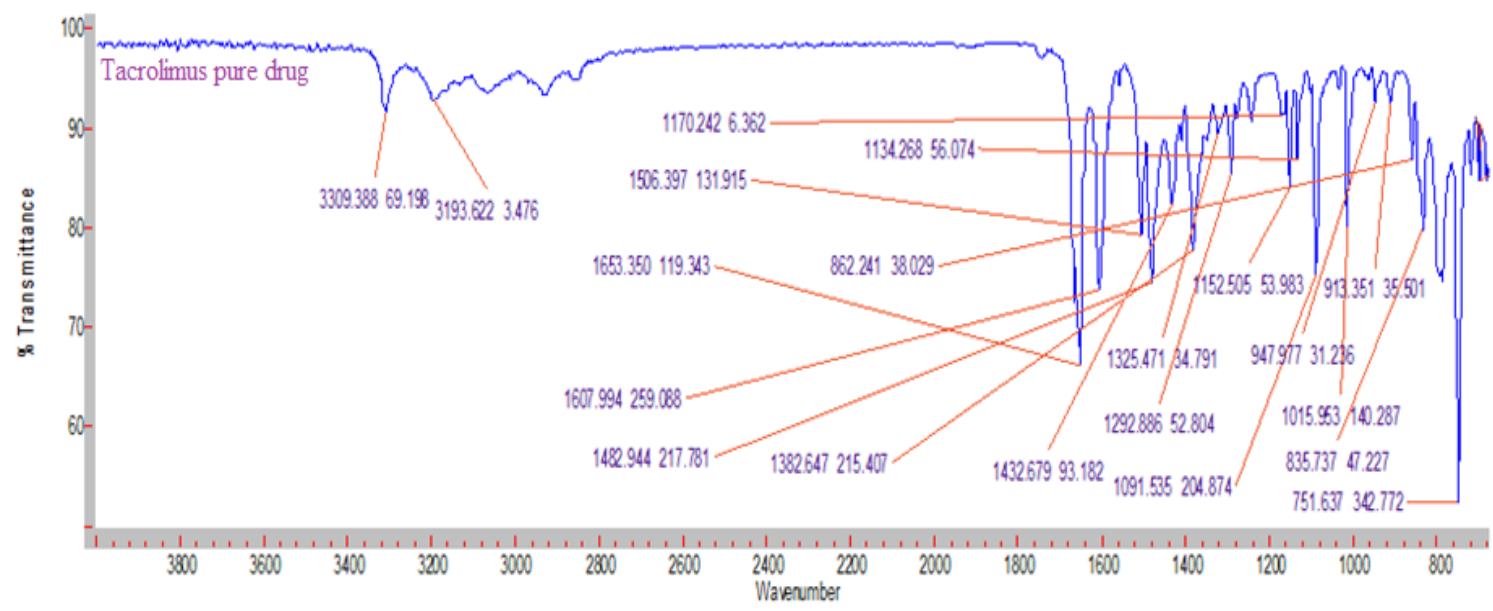

Figure 5a: FTIR spectrum of Tacrolimus pure drug

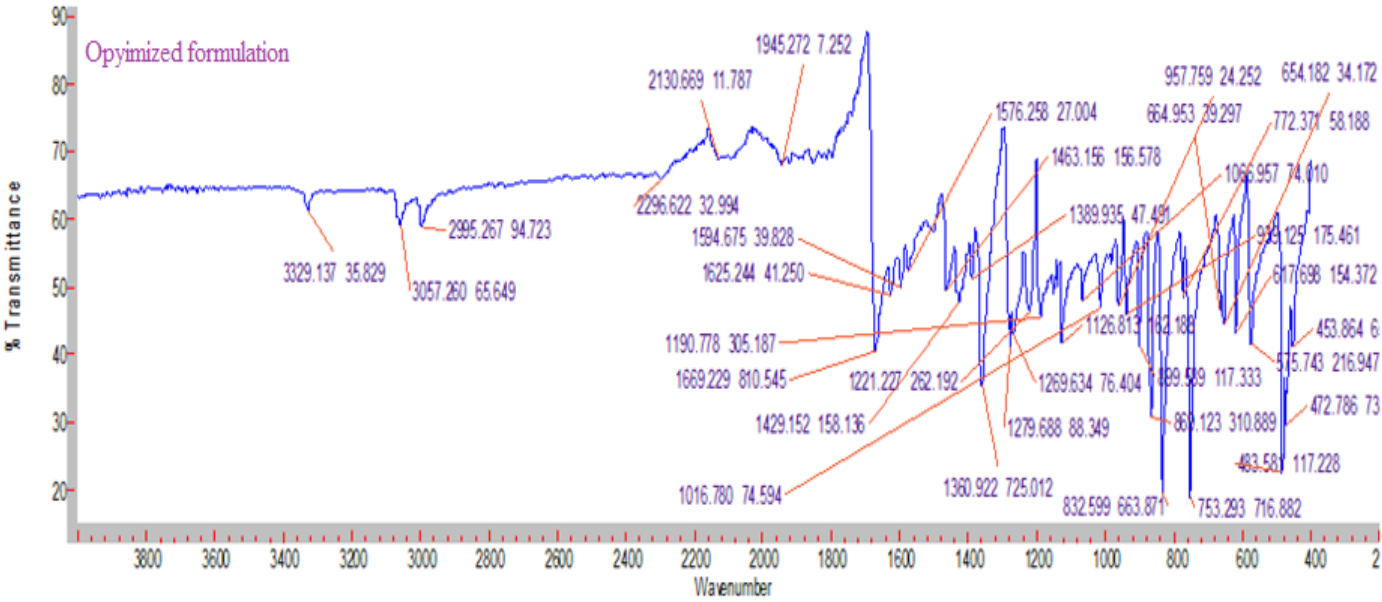

Figure 5b: FTIR spectrum of Tacrolimus optimised formulation TPN4

\section{Surface morphology}

The surface morphology of the pure drug, Maltodextrin and proniosome powders were examined by SEM and the images are represented in figure 6 . The absence of typical crystalline structures of tacrolimus in proniosome formulation indicates the transformation of drug to amorphous or molecular state. Further, the porous structure of maltodextrin as evident in figure was illegible in proniosome powders because of the deposition of surfactant and lipid on the surface of maltodxetrin.

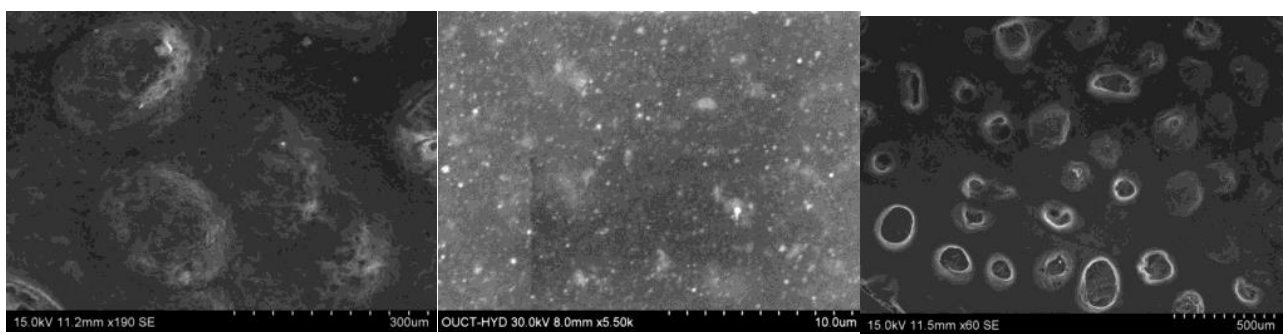

Figure 6: SEM picture of A) Maltodextrin B) Pure Drug C) TPN4 


\section{In situ perfusion study}

The in-situ perfusion study facilitates to ascertain the potential of proniosomes for improved absorption of tacrolimus across $\mathrm{GI}$ tract. The effective permeability coefficient (Peff), absorption rate constant (Ka) and enhancement ratio were calculated and represented in table 5. The obtained Peff values for control, TPN5, TPN7 and TPN4 were $1.21 \pm 0.6,3.20 \pm 1.3,5.41 \pm 1.3$ and $6.54 \pm 1.5 \mathrm{~cm} / \mathrm{sec}\left(\times 10^{-6}\right)$ respectively. The absorption rate constant $(\mathrm{Ka})$ indicative of rate of absorption was also significantly higher for proniosomes compared to control. The enhancement ratio above 1 indicates an enhanced permeation and in our case with all the proniosome formulations we could observe an ER greater than 1 which suggests the potential of proniosomes for improved oral delivery.

Table 5: In situ parameters of tacrlimus from optimized proliposome powders across rat intestine (Mean \pm SD; $\mathrm{n}=3$ ).

\begin{tabular}{lllll}
\hline Formulation & Peff $(\mathrm{rat})(\mathrm{cm} / \mathbf{s e c}) \times 10^{-6}$ & Fa (\%) & Ka (h-1) & ER \\
\hline Control & $1.21 \pm 0.6$ & $5.8 \pm 1.8$ & $0.065 \pm 0.001$ & - \\
TPN-5 & $3.20 \pm 1.3$ & $15.2 \pm 1.6$ & $0.022 \pm 0.001$ & $2.63 \pm 0.4$ \\
TPN-7 & $5.41 \pm 1.3$ & $22.3 \pm 5.6$ & $0.031 \pm 0.001$ & $4.26 \pm 1.3$ \\
TPN-4 & $6.54 \pm 1.5$ & $25.2 \pm 4.4$ & $0.032 \pm 0.002$ & $5.42 \pm 1.4$ \\
\hline
\end{tabular}

\subsection{Pharmacokinetic study}

The objective of the present study is to check the feasibility of tacrolimus loaded proniosomes to improve the oral bioavailability. It is speculated that the positive charge containing vesicles favour the drug absorption due to increased vesicle interaction with the cell surface because of electrostatic attraction. The mean serum concentration vs. time profiles of Tacrolimus following peroral administration of different proniosomes in comparison to control. The pertinent pharmacokinetic parameters were derived and presented in Table 6.

Table 6: Pharmacokinetic parameters of tacrolimus in rats following oral administration of proniosome powders (mean $\pm S D, n=6)$.

\begin{tabular}{lllll}
\hline $\begin{array}{l}\text { Pharmacokinetic } \\
\text { Parameters }\end{array}$ & \multicolumn{4}{l}{ Formulations } \\
\cline { 2 - 5 } & Control & TPN-7 & TPN-5 & TPN-4 \\
\hline Cmax $(\mu \mathrm{g} / \mathrm{ml})$ & $0.166 \pm 0.04$ & $0.42 \pm 0.01$ & $0.37 \pm 0.05$ & $0.55 \pm 0.06$ \\
$\operatorname{Tmax}(\mathrm{h})$ & $2.2 \pm 0.2$ & $4.2 \pm 0.06$ & $4.8 \pm 0.04$ & $4.65 \pm 0.08$ \\
$\mathrm{~T}_{1 / 2}(\mathrm{~h})$ & $17.14 \pm 2.38$ & $23.2 \pm 4.2$ & $28.12 \pm 3.42$ & $27.36 \pm 3.52$ \\
$\mathrm{~K}\left(\mathrm{~h}^{-1}\right)$ & $0.038 \pm 0.01$ & $0.032 \pm 0.01$ & $0.023 \pm 0.011$ & $0.024 \pm 0.012$ \\
$\mathrm{AUC}_{0-\infty}\left(\mu \mathrm{g} \mathrm{h} \mathrm{m}^{-1}\right)$ & $7.05 \pm 0.83$ & $12.81 \pm 1.12$ & $10.25 \pm 0.82$ & $19.96 \pm 1.33$ \\
$\mathrm{MRT}_{0-\infty}(\mathrm{h})$ & $9.65 \pm 1.41$ & $10.24 \pm 1.68$ & $9.96 \pm 1.26$ & $13.45 \pm 2.14$ \\
$\mathrm{~F}$ & $1 \pm 0$ & $1.46 \pm 0.24$ & $1.29 \pm 0.13$ & $2.34 \pm 0.16$ \\
\hline
\end{tabular}

The obtained results reveal a higher Cmax for proniosomes compared to control $(p<0.001)$. However, the time to reach the peak concentration (Tmax) remained constant from all the formulations. The higher biological half-life and mean residence time of tacrolimus from proniosomes with respect to control is obviously due to the slower elimination rate of tacrolimus from these formulations (Table 6). Based on the AUC and F values, the formulations can be ranked in the following descending order TPN4 > TPN7 > TPN5 > Control. The improved bioavailability of tacrolimus from proniosomes could be due to the contribution of several mechanisms either alone or in combination which include i) by virtue of the surfactant property of the phospholipids can reduce the interfacial barrier and provide intimate contact with epithelial cell membrane thus favouring the partitioning of tacrolimus into the hydrophobic domain of the cell membrane ii) fusion of the niosomes with the epithelial cells by endocytosis also might be responsible for the augment in absorption across GI membrane iii) direct transfer of niosomes 
formed at the vicinity of the GI tract may lead to an improved bioavailability due to avoidance of first pass metabolism.

\section{Stability study}

The physical appearance \% retention of tacrolimus was monitored for the optimized proniosome powder formulation (TPN4) upon storage at refrigerated and ambient room temperature for a period of 180 days. At definite time intervals, the proniosome powder was hydrated to form niosomes and we could observe the formation of vesicles without any signs of drug crystallization. The entrapment efficiency was also monitored and the results indicate that there was no appreciable change in the $\%$ retention of tacrolimus when stored at refrigerated temperature. In contrast, the formulation was destabilized at room temperature resulting in drug leakage with less entrapment efficiency with time. The stability studies suggest that the proniosome formulation was comparatively more stable when stored at refrigerated conditions compared to room temperature.

\section{Conclusion:}

In the present work, Proniosomal powder containing tacrolimus was prepared and evaluated. The optimized formulation typically contains span 60, cholesterol, maltodextrin and API. Formulated proniosomes gave satisfactory results for various evaluation parameters like particle size, Zetapotential, Powdered XRD, DSC, Scanning electron Microscopy, FTIR entrapment efficiency, In-vitro Dissolution and In-vivo/Ex-In-vivo. From this study, we concluded that solubility and bioavailabilty of tacrolimus can be improved by incorporating it into proniosomal system.

\section{References:}

1) Sudhamani, Proniosomes-A Promising Drug Carriers, International Journal PharmTech research, 2010.

2) Annakula D, Errabelli MR, Jukanti R, Bandari $S$, Veerareddy $\mathrm{P}$, Provesicular Drug Delivery Systems: An
Overview and Appraisal. Archives of Applied Science Research, 2(4), 2010, 135-46.

3) B. Bachchhao Kunal, S. Jaware Mayuri, K. Deshmukh Prashant, D. Patil Dipak and B. BariSanjay, UV Spectroscopic Method for Estimation of Tacrolimus in Bulk and Tablet Formulation by Area Under Curve Method, International Journal of Pharmaceutical and Chemical Sciences, 4 (3),2015, 326-329.

4) Raja K, Jestin Palu Ukken, Athul P V, Tamizharasi.S, Sivakumar.T, Formulation and Evaluation maltodextrin based proniosomal drug delivery system containing anti-diabetic (Glipizide) drug, International Journal of Pharm Tech Research, 2011, 471-477.

5) T.Sudhamani, V.Ganesan, N.Priyadarsiniand, M.Radhakrishnan, Formulation and evaluation of Ibuprofen loaded maltodextrin based Proniosome, International Journal of Biopharmaceutics, 2010, 7581.

6) Tamizharasi Sengodon, Biradar Sunil, Rathi Vaishali, Rathi Jagdish Chandra. "Formulation and Evaluation of indomethacin loaded maltodextrin based proniosomes". International journal of PharmTech Research, 1(3), 2009, 517-523.

7) Solanki A, Parikh J, Parikh R., Preparation, Characterization, Optimization, and Stability Studies of Aceclofenac Proniosomes, Iranian Journal of Pharmaceutical Research, 7(4), 2009, 237-246.

8) R. Jukanti, S. Sheela, S. Bandari, P.R. Veerareddy, Enhanced bioavailability of exemestane via proniosomes based transdermal delivery. Journal of Pharmaceutical Sciences, 100,2011, 3208-3222.

9) G. Hazel, D Akhilesh, P Prabhakara, V Kamath Jagannadh. Development and Evaluation of Norfloxacin loaded maltodextrin based proniosomes, International research journal of pharmacy, 3 (6), 2011, 176-179.

10) Rhodes DG, Blazek-Welsh, SEM Imaging Predicts Quality of Niosomes from Maltodextrins Based Proniosomes, Pharmaceutical Research, 18(5), 2001, 656-661.

11) Chongjin H, David GR, Proniosomes: A Novel Drug Carrier Preparation, International Journal of Pharmaceutics, 1999, 23-35.

12) Bairwa K, Deepika C, Proniosome A review, Asian Journal of Biochemical and Pharmaceutical Research, 1(2), 2011, 690-994. 


\section{*Corresponding Author:}

Pradeep Kumar Yennamameni

Email: pradppharma@gmail.com 